评 述 中国知名大学及研究院所专栏 中国科学技术大学生命科学学院专辑

\title{
表观遗传学研究进展
}

\author{
丁勇, 许超, 吴季辉, 施蕴渝", 藏建业, 蔡刚 \\ 中国科学技术大学生命科学学院, 合肥 230027 \\ * 联系人, E-mail: yyshi@ustc.edu.cn
}

收稿日期: 2016-10-30; 接受日期: 2016-12-02; 网络版发表日期: 2017-01-20

\begin{abstract}
摘要表观遗传学调控真核基因表达, 与人类重大疾病, 如肿瘤、神经退行性疾病、自身免疫性疾病等密切相 关, 一直以来都是生命科学研究领域的一个热点. 近年来人们在表观遗传学领域取得了相当丰硕的成果, 本文 将从表观遗传学修饰(含 RNA 表观遗传修饰)、染色质重塑和植物的表观遗传学三个方面总结中国科学技术大学 生命科学学院在表观遗传学领域的最新进展.
\end{abstract}

关键词表观遗传学, 组蛋白修饰, CXXC 结构域, royal family, DNA 甲基化, RNA 甲基化, 染色质重塑

表观遗传学是研究除 DNA 序列变化外的其他机 制引起的细胞表型和基因表达的可遗传的改变. 表 观遗传学现象涉及组蛋白修饰、DNA 甲基化、RNAi、 染色质重塑等. 表观遗传机制主要在基因转录层面 调控基因表达，最近新兴起的 RNA 表观遗传则以 N6-甲基化腺嘌呤为标志, 研究后转录过程对真核基 因表达的调控.

\section{1 表观遗传学修饰}

\section{1 组蛋白修饰}

在染色质的组蛋白上可以发生各种位点特异的 翻译后修饰, 如组蛋白甲基化、乙酰化、泛素化和磷 酸化等. 近年来又先后发现其他一些修饰, 如 Sumo 化、ADP ribosylation、巴豆酰化等. 不同的组蛋白修 饰通过不同的作用机制调控染色质的结构和功能. 相应于各种组蛋白修饰有各种酶类, 建立修饰的酶,
被称为“Writer’，如甲基转移酶、乙酰化转移酶等. 而 去除修饰的酶被称为 ‘Eraser’，如去甲基化酶、去乙酰 化酶等. 组蛋白翻译后修饰能够募集一些效应分子, 这些效应分子能识别组蛋白翻译后修饰, 然后引起 下游功能改变，这些效应分子被称为“Reader’。下面 就赖氨酸乙酰化、赖氨酸甲基化以及精氨酸甲基化这 几种主要的组蛋白修饰为例来描述一下这些效应蛋 白质识别及调控组蛋白修饰的生物学过程.

(1) 组蛋白赖氨酸乙酰化识别及调控. 组蛋白的 乙酰化可以发生在人组蛋白 $\mathrm{H} 3(\mathrm{~K} 4, \mathrm{~K} 9, \mathrm{~K} 14, \mathrm{~K} 18$, K23, K27, K36, K56 和 K79), H4 (K5, K8, K12, K16, $\mathrm{K} 20$ 和 $\mathrm{K} 91), \mathrm{H} 2 \mathrm{~A}(\mathrm{~K} 5$ 和 $\mathrm{K} 9)$ 和 $\mathrm{H} 2 \mathrm{~B}(\mathrm{~K} 5, \mathrm{~K} 12$, $\mathrm{K} 15, \mathrm{~K} 16, \mathrm{~K} 20$ 和 K120). 乙酰化修饰的范围比甲基 化修饰的范围更广，一般来讲，这个修饰总是跟转录 激活相关，赖氨酸乙酰化导致了赖氨酸正电性质的 改变, 使得染色质的结构更加开放, 导致基因转录更 加易于进行.

引用格式: 丁勇, 许超, 吴季辉, 等. 表观遗传学研究进展. 中国科学: 生命科学, 2017, 47: 3-15

Ding Y, Xu C, Wu J H, et al. Recent progress in epigenetics. Sci Sin Vitae, 2017, 47: 3-15, doi: 10.1360/N052016-00317 
赖氨酸乙酰化是由组蛋白乙酰转移酶, 以乙酰 辅酶 $\mathrm{A}$ 为供体, 将乙酰基团转移到赖氨酸的 $\varepsilon$ 基上实 现的. 组蛋白乙酰基团也可以被一类称为组蛋白去 乙酰化酶所去除, 因而整体来讲组蛋白的乙酰化水 平处于这两类酶的动态调节中. 组蛋白上被乙酰化 修饰的赖氨酸被效应分子识别将信号传给下游分子.

MOZ 和 MORF 是两个对造血干细胞和神经干细 胞的发育有重要影响的组蛋白乙酰转移酶, 并调节 HOX 基因的表达. 在白血病中, 它们的基因往往发 生染色体异位. 在细胞内 MOZ/MORF 与 ING5, EAF6, BRPF $1 / 2 / 3$ 形成复合物, 其中 BRPF 蛋白起着 桥梁的作用, 促进转录, 并能增强 $\mathrm{MOZ}$ 的乙酰转移 酶活性 ${ }^{[1]}$. 我们报告了人 $\mathrm{MOZ}$ 串联 PHD 结构域 (PHD12)处于自由状态的溶液结构, 以及和 H3K14ac 肽 $1.47 \mathrm{~A}$ 复合物的晶体结构, 揭示了该串联结构域与 乙酰化组蛋白 H3 未修饰的第二位精氨酸(H3R2), 以 及乙酰化的第 14 位的赖氨酸 (H3 K14ac)识别的结构 基础 (图 1A). 此外, 染色质免疫沉淀 (ChIP) 和 RT-PCR 分析的结果表明, PHD12 与组蛋白的识别将 有助于将 $\mathrm{MOZ}$ 复合物定位到 HOXA9 基因的启动子 位点上, 从而促进 HOXA9 基因在启动子区域 $\mathrm{H} 3$ 的乙 酰化, 进而上调 HOXA9 mRNA 的水平. 研究表明, 通过 PHD12 的识别 H3R2/K14ac, 是一种重要的表观 遗传调控机制 ${ }^{[2]}$. 最近, 清华大学李海涛实验室发现, MOZ 的 PHD12 还可以以更强的方式结合巴豆酰化的 $\mathrm{H} 3 \mathrm{~K} 14^{[3]}$. BRPF 蛋白中含有两个 PHD 锌指、一个
Bromodomain、一个 PWWP 结构域. 通过实验证明, BRPF2-PHD1 锌指结构域, 能特异结合非甲基化的 组蛋白 $\mathrm{H} 3 \mathrm{~K} 4$, 但不能结合甲基化的组蛋白 H3K4. 我们用核磁共振方法解析了 BRPF2-PHD1 与组蛋白 $\mathrm{H} 3 \mathrm{~K} 4 \mathrm{me} 0$ 小肽复合物的溶液结构 ${ }^{[4]}$. 染色质免疫共 沉淀(chromatin immuno precipitation, CHIP) 实验表 明, BRPF2-PHD1 与 $\mathrm{H} 3 \mathrm{~K} 4 \mathrm{me} 0$ 的相互作用与 $\mathrm{BRPF} 2$ 在 HOXA9 基因座的定位有关. 我们还测定了 BRPF2PHD2 与 DNA 复合物的结构, 揭示其功能意义 ${ }^{[5]}$.

Bromodomain 是一类与乙酰化组蛋白特异识别 的蛋白质结构域。我们测定了人的系列含 Bromodomain 的蛋白质, 如 Brg1, Brd2, Brd4, Brd7 的 Bromodomain 的三维结构, 分别研究了它们与乙酰 化组蛋白的特异识别 ${ }^{[6-9]}$. 其中, Brg1 是人的染色质 重塑复合物 SWI/SNF 复合物的核心催化亚基, 在细 胞周期调控、细胞分化和肿瘤发生中发挥重要作用. 我们测定了 Brg1 的 Bromodomain 的三维结构, 发现 Brg1 的 Bromodomain 与组蛋白特定位点乙酰化的赖 氨酸的特异识别, 并阐明了二者识别方式, 及关键残 基 $^{[6]}$. Brd2, Brd4 是 BET 家族成员, Brd7 也是染色质 重塑复合物的重要组分, 目前这些 Bromodomain已 经成为重要的药物作用靶点. 阮科副教授在中国科 学技术大学生命科学学院建立了国内首个基于核磁 共振方法的片段药物篮选与鉴定平台, 以 Bromodomain 及其他表观遗传相关靶标为对象, 已 篮选并在结构的基础上优化设计了多个抗肿瘤增殖
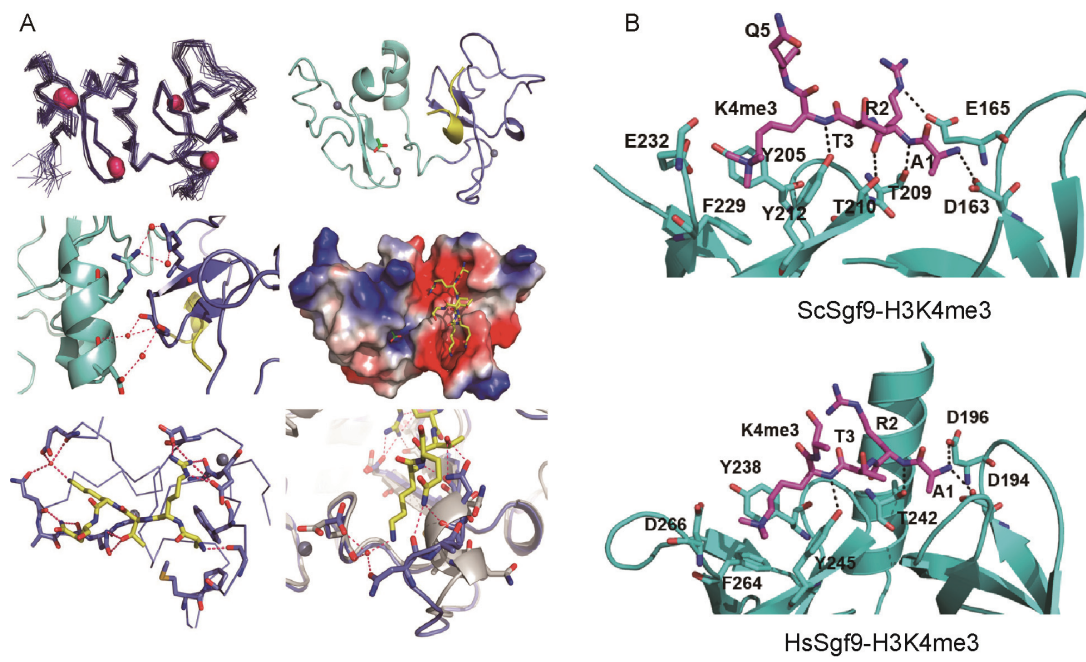

图 1 MOZ PHD12 结构及其对 H3R2, H3K14ac 的特异识别(A)与酿酒酵母和人的 Sgf29 的 tandem Tudor 结构域与多肽 H3K4me3 的复合物结构 $(B)$ 
和迁移的靶点的小分子抑制剂. 初步结果已经发表. 还针对基于片段的药物发现过程中，苗头化合物和 靶点蛋白的弱相互作用难以表征的瓶颈问题, 发展 了转移顺磁弛豫增强技术. 并发表了特邀综述, 申请 了专利, 有望开发成具有我国自主产权的药物先导 化合物.

（2）组蛋白赖氨酸甲基化识别及调控. (i ) 组 蛋白赖氨酸甲基化识别. 核心组蛋白 H3 和 H4 上有 多个赖氨酸位点能被甲基化, 包括组蛋白 $\mathrm{H} 3 \mathrm{~K} 4$, H3K9, H3K27, H3K36, H3K79 和 H4K20 ${ }^{[10]}$. 甲基化 组蛋白赖氨酸能被多种识别结构域识别, 在染色质 高级结构控制、基因转录调控、DNA 损伤修复、RNA 剪切等过程中起着重要的作用 ${ }^{[11]}$. 例如在基因转录 调控过程中, 通常认为甲基化组蛋白 $\mathrm{H} 3 \mathrm{~K} 4, \mathrm{H} 3 \mathrm{~K} 36$ 和 H3K79 与转录激活相关, 而 H3K9, H3K27 和 H4K20 被认为与转录抑制有关. 组蛋白赖氨酸的甲 基化还能调控组蛋白的其他翻译后修饰(如乙酰化 等), 也能被其他组蛋白修饰(如泛素化)调控 ${ }^{[2,13]}$.

目前, 已经发现多种甲基化组蛋白赖氨酸的识 别结构域, 包括 PHD 结构域 ${ }^{[14]}$,WD40-repeat 结构 域 ${ }^{[15]}$ 、Ankyrin-repeat 结构域 ${ }^{[16]}$ 和 Royal 结构域家族 蛋白等. Royal 结构域家族包括 Tudor 结构域、Chromo 结构域、MBT 结构域、PWWP 结构域等 ${ }^{[17]}$. 虽然甲 基化组蛋白赖氨酸识别结构域之间的氨基酸序列 和空间结构差异较大, 其甲基化赖氨酸识别位点都 是由芳香族氨基酸组成的“aromatic cage”, 结构特征 相似 ${ }^{[17,18]}$.

Tudor 结构域是一类重要的甲基化组蛋白赖氨酸 识别结构域, 在基因转录中起着重要的作用. 酿酒酵 母 SAGA 复合物是由多个亚基组成的分子量为 1.8 $\mathrm{MDa}$ 的转录共激活复合物, 包括乙酰转移酶 $\mathrm{Gcn} 5$ 和 去泛素化酶 Ubp8, 能使组蛋白乙酰化和去泛素化, 在基因表达过程中起着激活基因转录的作用 ${ }^{[19]}$. 研 究发现, SAGA 复合物的亚基 Sgf29 羧基端具有两个 串联的 Tudor 结构域, 能识别甲基化修饰的组蛋白

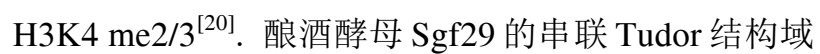
采取面对面排列, 与其他已知的串联 Tudor 结构的空 间折叠模式有较大的差别. 在 Sgf 29 吕联 Tudor 结构 与组蛋白 H3K4 me $2 / 3$ 多肽的结构中, 第一个 Tudor 结构域有一个带负电的小结合口袋，负责结合 $\mathrm{H} 3 \mathrm{~A} 1$; 第二个 Tudor 结构域具有由芳香族氨基酸组成的甲 基化赖氨结合口袋, $\mathrm{H} 3 \mathrm{~K} 4$ 的甲基化赖氨酸侧链插入
其中(图 1B). Sgf29 羧基端串联 Tudor 结构域的结构 特征及其与甲基化组蛋白赖氨酸多肽相关作用的模 式, 决定了其特异性地识别组蛋白 $\mathrm{H} 3 \mathrm{~K} 4 \mathrm{me} 2 / 3$. Sgf 29 与组蛋白 H3K4 me2/3 的相互作用控制着 SAGA 复合物在染色质上的募集, 并能影响组蛋白乙 酰化水平, 从而调控基因转录水平 ${ }^{[20]}$.

(ii) 组蛋白赖氨酸甲基化/去甲基化调控. 目前 发现的组蛋白赖氨酸去甲基化可以分为两大类, 即 胺氧化酶家族和含有 $\mathrm{JmjC}$ 结构域的氧化酶家族 ${ }^{[21,22]}$. 由于化学反应机理的差异, 胺氧化酶家族去甲基化 酶仅能催化单甲基化和二甲基化的赖氨酸去甲基化, 而 JmjC 家族去甲基化酶能催化单甲基化、二甲基化 及三甲基化修饰的去甲基化 ${ }^{[23]}$. 已经发现的含有 $\mathrm{JmjC}$ 结构域的组蛋白去甲基化酶可以分为 7 个亚家 族: KDM2, KDM3, KDM4, KDM5, KDM6, JHDM1 及 JmjC domain only ${ }^{[24,25]}$. 这些组蛋白去甲基化酶能 调控基因转录、染色质着丝粒区域的组装、DNA 损 伤修复等, 其功能异常将导致肿瘤、精神发育异常等 重大疾病的发生 ${ }^{[24]}$.

对于一些仅含有 $\mathrm{JmjC}$ 结构域的蛋白, 其底物特 异性还存在争议. 例如, 胞内实验表明, NO66 具有 H3K $4 m e$ 和 H3K36me 的去甲基化酶活性, 能与骨细 胞分化过程中的关键转录因子 Osx 相互作用, 调节成 骨细胞的发育以及骨量维持 ${ }^{[26,27]}$; 而又有体外的生 化研究表明, NO66 与 Mina53 作为典型的仅含有 $\mathrm{JmjC}$ 结构域酶类，能分别羟基化核糖体亚基 $\mathrm{Rp} 18$ 和 $\operatorname{Rp} 127 \mathrm{a}^{[28,29]}$.

我们测定了人源 NO66 的结构, 发现 NO66 单体 的结构中含有保守的 $\mathrm{JmjC}$ 结构域, 钩状结构域和 wHTH 类似的结构域, 4 个 NO66 分子能形成环状四 聚体 ${ }^{[27]}$ (图 2A 和 B). 我们鉴定了 Osx 与 NO66 相互 作用区域的氨基酸序列，发现了NO66 四聚体分子的 表面与 Osx 相互作用的界面(图 2C), 破坏 NO66 与 Osx 的相互作用将导致组蛋白 H3K4 甲基化水平偏 高, Osx 依赖的骨骼发育关键基因表达水平升高, 促 使骨细胞分化加速、骨骼发育异常 ${ }^{[27,30]}$. 氨基酸序列 同源性分析发现, NO66 与 Osx 相互作用的界面在脊 椎动物中是保守的, 提示 NO66 与 Osx 相互作用调控 骨细胞分化的机制在进化上是保守的 ${ }^{[27]}$. 我们还测 定了 NO66 与 Rp18 多肽(AA204 224)的复合物结构, 发现 NO66 与 Rp18 $8^{204 \sim 224}$ 多肽中 N215, H216 和 H218 形成氢键相互作用, 决定了其底物特异性(图 2D) ${ }^{[28]}$. 

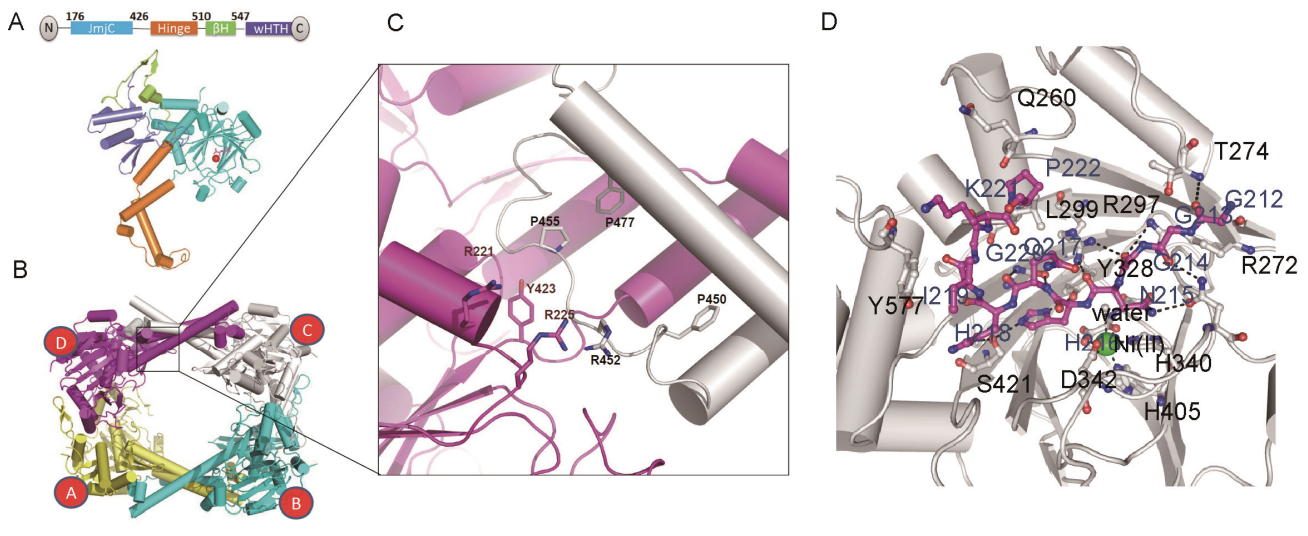

图 2 NO66 识别转录因子 Osx 和底物的结构基础

A: NO66 单个亚基的结构; B: NO66 四聚体结构; C: NO66 结合转录因子 Osx 位点位于 NO66 四聚体结合界面上; D: NO66 识别着基化底物 Rpl8 多肽的分子机制

研究提示, NO66 可能是一类具有组蛋白去甲基化和 蛋白质羟基化作用的双功能酶.

（3）组蛋白精氨酸甲基化调控及识别. 精氨酸甲 基化在基因表达调控，RNA 代谢，细胞信号转导及 DNA 修复等过程中发挥重要作用 ${ }^{[31 \sim 33]}$. 这个过程是 由精氨酸甲基转移酶(PRMT)催化的, 以腺苷甲硫氨 酸(AdoMet) 为甲基供体, 生成腺苷高半胱氨酸 (AdoHcy)和甲基化精氨酸. 根据甲基化精氨酸产物 的不同，非对称二甲基精氨酸，对称二甲基精氨酸， 及单甲基精氨酸, 精氨酸甲基转移酶被相应划分为 I 类, II 类和III类. 我们解析了布氏雉虫精氨酸甲基 转移酶 $T b P R M T 7$ 与 AdoHcy 及 $\mathrm{H} 4$ 小肽底物三元复 合物的三维结构, 这是第三类精氨酸甲基转移酶的 第一个三维结构, 同时也是第二个解析的精氨酸甲 基转移酶-AdoHcy-小肽底物三元复合物 ${ }^{[34]}$. 揭示了 它作为第三类精氨酸甲基转移酶, 具有严格的催化 精氨酸单甲基化活性的结构基础(图 3). 还解析了 TbPRMT6 和 TbPRMT6-AdoHcy 二元复合物的结 构 $^{[35]}$, 发现 AdoHcy 的结合引起 TbPRMT6 活性位点 附近的构象调整. 免疫印迹和质谱学结果表明, TbPRMT6 在体外可以对称性二甲基化牛组蛋白 H4R3，但是不能催化雉虫组蛋白的小肽.

目前已知 Tudor 结构域可以特异识别甲基化精 氨酸, 其识别方式与以上甲基化赖氨酸的识别方式 极其相似, 也是通过一个芳香环口袋识别甲基化精 氨酸的甲基 ${ }^{[36]}$.

\section{$1.2 \mathrm{CpG}$ 结合蛋白质与 DNA 甲基化的调控}

除组蛋白修饰外, DNA 甲基化 $\left(\mathrm{m}^{5} \mathrm{C}\right.$ 或 $\left.\mathrm{mC}\right)$ 也参 与调控基因转录, 主要表现为抑制基因表达 ${ }^{[37]}$. 绝大 部分 DNA 甲基化发生在胞嘧啶-鸟嘌呤 $(\mathrm{CpG})$ 双核苷 的胞嘧啶上. 已知 $\mathrm{CXXC}^{[38]}$ 和 $\mathrm{MBD}^{[37]}$ 结构域分别结 合非修饰和甲基化的 CpG. CXXC 为 40 60 个氨基酸 长的锌指结构域, 因含有两个 CXXCXXC 序列而得 名 ${ }^{[39]}$. 人有十余种含 CXXC 结构域的蛋白质, 多是 表观遗传学调控相关的酶 ${ }^{[39]}$. 我们在 2010 年解析了 CFP1 的 CXXC 结构域与 CpG 双链 DNA 的晶体结构, 发现 CFP1 的 CXXC 结构域利用含有正电的表面接触 带负电的 DNA 的磷酸主链 ${ }^{[40]}$. CXXC 结构域与 $\mathrm{CpG}$ 碱基对形成多个主链氢键, 由此揭示了 CXXC 结构 域特异识别 $\mathrm{CpG}$ 双链 DNA 的结构机理(图 4A) ${ }^{[40]}$. CFP1 通过 CXXC 结合 CpG DNA 后, 抑制该区域的 $\mathrm{CpG}$ 甲基化, 并募集 SETD1A 复合物, 产生 $\mathrm{H} 3 \mathrm{~K} 4$ 甲 基化, 从而激活基因转录 ${ }^{[41]}$.

我们发现 CXXC 家族成员尽管其结构相似, DNA 结合特性却不尽相同. 例如 CFP1 的 CXXC 偏 好 $\mathrm{CpGG}$ 三核苷序列, 有更强的序列选择性 ${ }^{[40]}$. 还解 析了爪蟾 Tet3 CXXC 结构域与不同双链 DNA 的复合 物结构. Tet 3 催化甲基化胞嘧啶 $(\mathrm{mC})$ 羟甲基化, 从而 导致 DNA 去甲基化 ${ }^{[42]}$. 我们发现 Tet3 的 CXXC 具有 更广泛的 DNA 结合能力, 不仅结合非修饰 $\mathrm{CpG}$, 还 可以借助一个非修饰的胞嘧啶(C) $(\mathrm{CpA}, \mathrm{CpT}$ 或 $\mathrm{CpC})$ 结合甲基化 $\mathrm{CpG}(\mathrm{mCpG})\left(\right.$ 图 4B) ${ }^{[42]}$. 由此构建了 Tet3 的 CXXC 与其酶活结构域协同作用的模型: Tet 3 通过 $\mathrm{CXXC}$ 结合 $\mathrm{C}$, 从而募集在富含 $\mathrm{mCpG}$ 的区域, 如靶 

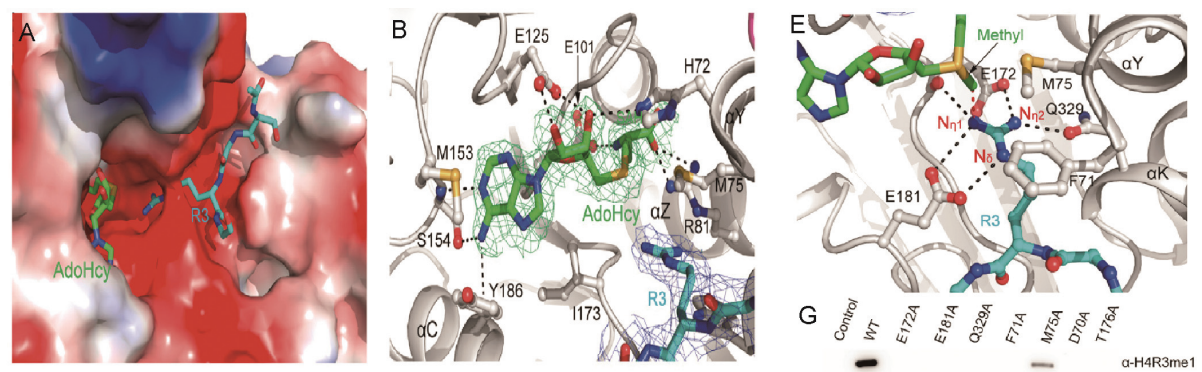

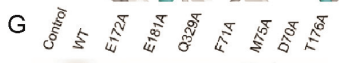
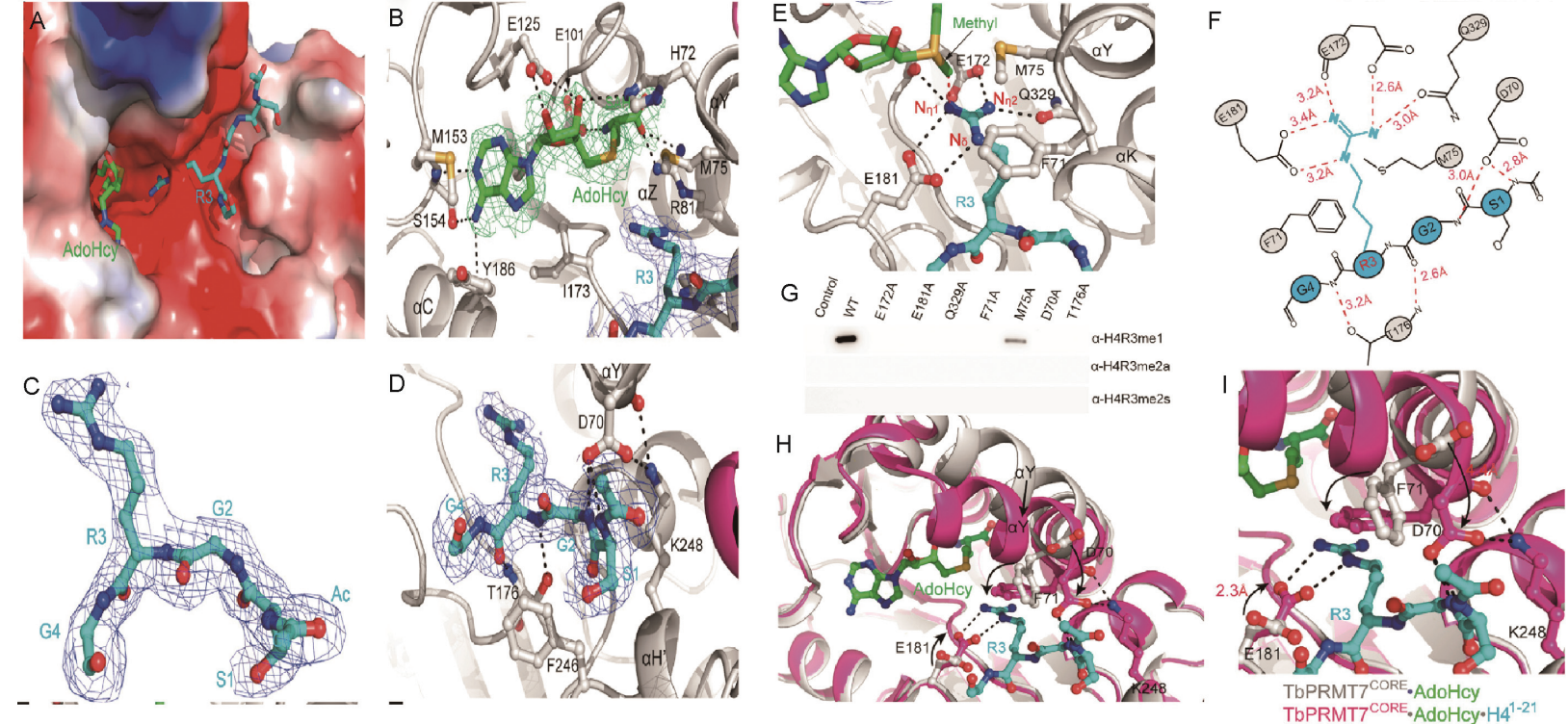

图 3 TbPRMT7-AdoHcy-小肽底物三元复合物

(A) 底物 $\mathrm{H} 4^{1-21}$ 结合及 AdoHcy 识别的概貌, 显示 TbPRMT7 $7^{\mathrm{CORE}}$ 的静电表面、正电势(蓝)、负电势(红)、电中性(白); (B) 对 AdoHcy 识别 的细节, 氢键以虚线表示; (C) 结合态 H4(1-21)的构象; (D) 与 H4(1-21)的相互作用细节; (E) 与残基 R3 侧链结合的 H4 $4^{1-21}$, TbPRMT7 中 涉及结合的残基予以显示和标注, 氢键用虚线表示; (F) H4 ${ }^{1-21}$ (青色)与 TbPRMT7(灰色)的结合; (G) 在 TbPRMT7 中参与和 $\mathrm{H} 4^{1-21}$ 结合的 残基突变后极大减少了酶活; (H) TbPRMT $7^{\mathrm{CORE}} \cdot \mathrm{AdoHcy} \cdot \mathrm{H} 4^{1-21}$ (品红色)与 TbPRMT7 $7^{\mathrm{CORE}} \cdot \mathrm{AdoHcy}$ (灰色)活性中心的比较; (I) 图 $4 \mathrm{H}$ 放大图

A
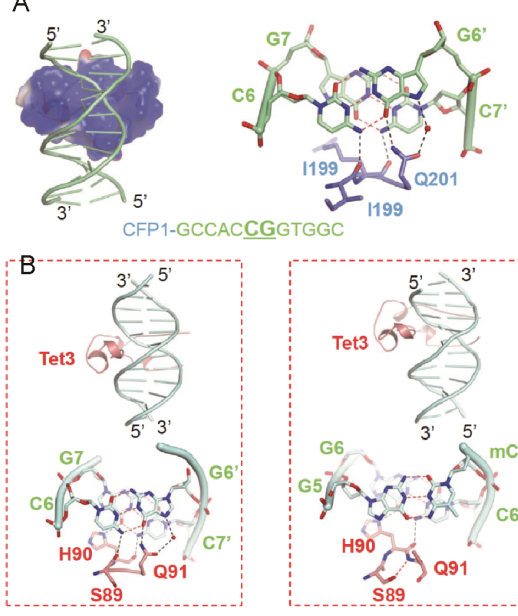

Tet3-GCCAACGTTGGC

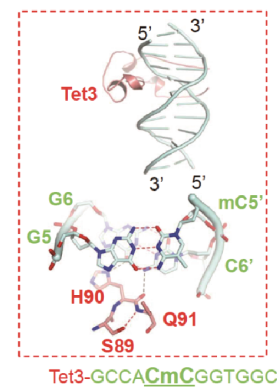

c

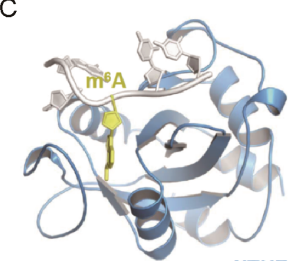

YTHDC1-GG(m6)

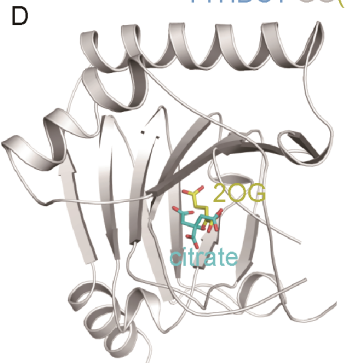

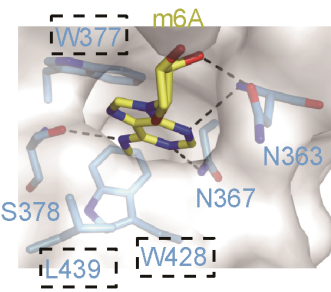

图 4 非修饰 CpG DNA 的识别、m6A RNA 的识别及消除机制

A: CFP1 的 CXXC 结构域特异性识别双链 DNA 的 CpG 序列; B: 爪蟾 Tet3 的 CXXC 结构域构象通变化分别识别 CpG 和 CmC; C: THDC1 的 YTH 结构域特异性识别单链 RNA 中的 $\mathrm{m}^{6} \mathrm{~A}$; D: ALKBH5-2OG 的复合物以及 ALKBH5 和 Citrate 的复合物叠合

基因的上游, Tet3 启动该区域 $\mathrm{mCpG}$ 的去甲基化, 使 得原来被抑制的基因被激活表达 ${ }^{[42]}$. 胞内实验表明, Tet3 CXXC 结构域缺陷将导致爪蟾胚胎发育早期神 经无法正常形成, 更严重导致眼睛缺失 ${ }^{[42]}$.
1.3 RNA 甲基化：RNA 甲基化识别与去甲基化的 机制

RNA 中存在上百种修饰，其中很多 RNA 修饰的 功能被不断揭示出来 ${ }^{[43]}$. N6-甲基化腺苷 $\left(\mathrm{m}^{6} \mathrm{~A}\right)$ 作为 
真核 mRNA 最丰富的内部修饰, 通过参与调控 mRNA 的剪切、定位、转运、稳定性及翻译等功能, 在 后转录层面参与真核基因表达调控 ${ }^{[44]} \cdot \mathrm{m}^{6} \mathrm{~A}$ 在体内受 到 3 种效应蛋白的调控, 书写器 (writer)、橡皮擦 (eraser) 和阅读器 (reader) ${ }^{[44]} \cdot \mathrm{m}^{6} \mathrm{~A}$ 甲基化转移酶是 METTL3-METTL14 复合物 ${ }^{[5 \sim 47]} ; \mathrm{m}^{6} \mathrm{~A}$ 去甲基化酶有

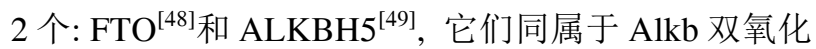
酶家族; $\mathrm{m}^{6} \mathrm{~A}$ 阅读器是 $\mathrm{YTH}$ 结构域 ${ }^{[0,51]}$.

不同的研究组先后发现 YTH 结构域结合 $\mathrm{m}^{6} \mathrm{~A}$ $\mathrm{RNA}^{[45,50]}$, 但是其作用机制未知. 包括本实验室在内 的研究组解析了多个 YTH 结构域与 RNA 的复合物 结构 ${ }^{[52 ~ 54]}$, 发现 YTH 结构域用带正电荷的表面结合 RNA, $m^{6} \mathrm{~A}$ 则插入 $\mathrm{YTH}$ 结构域的一个含有芳香环和 疏水氨基酸的口袋中 ${ }^{[52]} \cdot \mathrm{m}^{6} \mathrm{~A}$ 的甲基和芳环的 $\mathrm{pi}$ 电子 以及疏水氨基酸侧链之间的相互作用构成了 $\mathrm{m}^{6} \mathrm{~A}$ 特 异性识别的分子基基础(图 4C) ${ }^{[52]}$. 又解析了其他人 和酵母的 YTH 复合物结构, 揭示了这一 $\mathrm{m}^{6} \mathrm{~A}$ 识别模 式不但在人中, 而且在真核生物中都是保守的 ${ }^{[55]}$.

作为 RNA 去甲基化酶, ALKBH5 特异催化单链 $\mathrm{m}^{6} \mathrm{~A} \mathrm{RNA}^{[56]}$. 通过解析 ALKBH5 和 $\alpha$-酮戊二酸 (2OG) 以及锰的复合物结构, 揭示了 ALKBH5 结合 $20 G$ 和锰的位点, 并发现其内部的一个二硫键使得 ALKBH5 仅能识别单链, 而非双链 $\mathrm{RNA}^{[57]}$. 此外, 还通过模型，找到了 ALKBH5 中的两个底物结合残 基, 并发现突变两个中任何一个, 都会导致酶活丧 失 ${ }^{[57]}$. 更进一步地, 解析了 ALKBH5 和柠檬酸的复 合物, 发现柠檬酸占据了 ALKBH5 的 2OG 结合位点, 所以是 ALKBH5 的天然抑制剂(图 4D $)^{[57]}$. 这些复合 物结构为将来设计 ALKBH5 的小分子抑制剂提供了 方向和分子基础.

\section{2 染色质重塑}

\section{1 染色质重塑复合物}

ATP 依赖的染色质重塑复合物 SWI2/SNF2 超家 族 ${ }^{[58]}$, 其成员包括 $\mathrm{SWI} / \mathrm{SNF}$ (switch/sucrose nonfermentable), CHD(chromodomain helicase DNA-binding), ISWI(imitation switch)以及 INO80 (inositol- requiring80) 四类(图 5). 大量研究表明, 染色质重塑复合物参与 细胞核内包括基因表达调控、DNA 复制及染色质重 塑等众多生物学过程, 但是至今, 对其催化核小体重 塑的分子机制的认识还非常模糊 ${ }^{[59 ~ 61]}$.

\section{$2.2 \mathrm{SWI} / \mathrm{SNF}$ 家族染色质重塑复合物}

SWI/SNF 家族的染色质重塑复合体是最重要的 一类 ATP 依赖性的染色质重塑复合体, 主要包括 SWI/SNF 和 RSC 复合体. SWI/SNF 复合体可以通过 改变核小体的位置和密度来影响 DNA 结合蛋白接触 结合 DNA 的位点, 通过识别核小体组蛋白的尾部修 饰或者其他 DNA 结合蛋白, 导向特定的核小体, 通 过水解 ATP 可以通过以下多种途径改变核小体的结 构(改变核小体结合 DNA 位置, 驱逐组蛋白八聚体, 展开部分 DNA 片段或者驱逐部分组蛋白二聚体), 从 而暴露额外的 DNA 结合位点. DNA 依赖性的 ATPase 活性的亚基 Swi2/Snf2 负责水解 ATP 并且转移 DNA 的位置是 SWI/SNF 复合体的催化核心. Arp7 和 Arp9 是肌动蛋白相关的蛋白质亚基, 同时存在于 SWI/SNF 和 RSC 复合体中 ${ }^{[62]}$. 其中 Swi2/Arp7/Arp9 可以成稳定具有大多 SWI/SNF 生物学功能的核心亚 复合体 ${ }^{[63]}$. Snf5, Swi1, Snf6 及 Swi3 负责与转录因子 相互作用负责体内招募 SWI/SNF 复合体 ${ }^{[64,65]}$. Swp29/ Taf14 亚基不仅存在于 SWI/SNF 复合体，而且是基
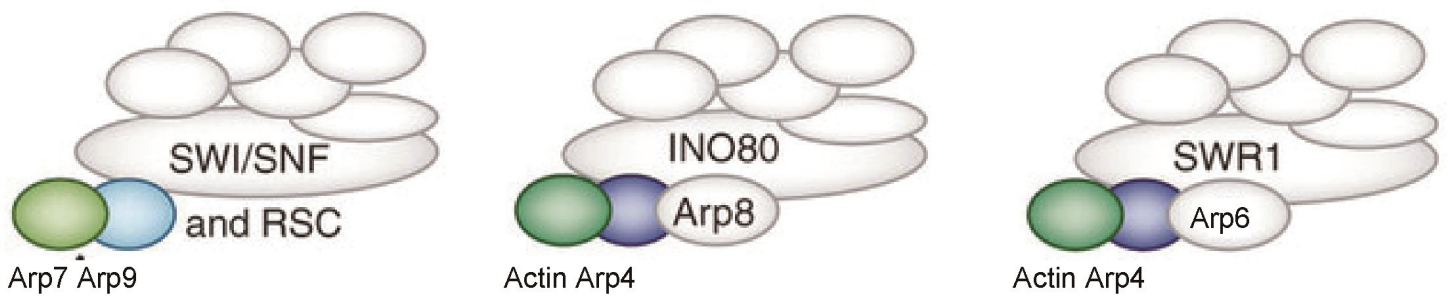

图 5 染色质重塑复合体 SWI/SNF, INO80, SWR1 及其 Actin/Arp 模块 
本转录因子 TFIIF 和 TFIID 复合体的组成亚基.

大量的遗传和生化研究表明, SWI/SNF复合体在 基因表达调控, 细胞周期发展, DNA 复制、重组和修 复都发挥重要作用 ${ }^{[62,66]}$. 哺乳类动物的 SWI/SNF 复 合体在胚胎干细胞分化过程中, 发生组成上的显著 变化, 暗示 SWI/SNF 复合体在胚胎干细胞分化过程 中发挥作用 ${ }^{[66]}$. 此外, 近年来的研究表明, SWI/SNF 复合体多个亚基的突变与多种癌症直接相关 ${ }^{[67 ~ 69]}$ ( 20\%的癌症对应多个 SWI/SNF 亚基的突变, 尤其是 SMARCA4/BRG1(Snf2), ARID1A/BAF250a(SWI1)和 SMARCB1/INI1(SNF5)等).

阐明各个亚基特定的生物学功能及其与核小体 之间的相互作用是阐明 SWI/SNF 复合体改变调控染 色质结构的分子机制所必须的, 然而目前为止, 对 SWI/SNF 各个亚基的特定的生物学功能的认识还非 常有限. SWI/SNF 家族复合体在体内的表达量非常 低, 组成庞大复杂(十几个亚基组成, 分子量>1 MDa) 而且还存在动态的亚基组成和构象多样性; 给 SWI/SNF 复合体的纯化和结构研究带来巨大挑战 ${ }^{[70]}$. 我们克服了重重困难, 已解析了 $\mathrm{Swi} / \mathrm{Snf}$ 复合体纳米 分辨率的冷冻电子显微镜结构及其重塑核小体的多 种中间状态的三维结构, 阐明了 Swi/Snf 的功能模块 和亚基组成, 为全面阐释 Swi/Snf 复合体重塑染色质 的分子机制打下了坚实基础(未发表资料).

\subsection{INO80 家族染色质重塑复合物}

INO80 和 SWR1 复合体同属于 INO80 家族成员, 都是十几个亚基的蛋白质复合物, 具有非常相似的 亚基组成和高度保守的结构域 ${ }^{[71,72]}$. 二者的催化亚 基 Ino80 和 Swr1 在其 ATPase 结构域都存在一个长的 插入, 用于招募共用的 Rvb1/2 解旋酶; 在其 $\mathrm{N}$ 端都 存在 HSA 结构域(helicase SANT-associated domain), 用于招募和结合肌动蛋白 (actin) 和肌动相关蛋白 (actin-related proteins, Arp); 两个复合体还共享了多 个亚基, 包括 RvB1, RvB2, actin, Arp4 等. INO80 和 SWR1 复合体高度保守的结构域、共用的多个亚基; 暗示着二者的结构应该高度相似. 出乎意料的是, 近 期在同期 Cell 杂志上发表的解析 INO80 和 SWR1 复 合体的电子显微镜结构却差异显著 ${ }^{[73,74]}$. 最显著的 不同有以下三个方面: SWR1 的构象非常紧致, 仅包 含单层 6 元杂环的 RvB 模块, Arp4-actin 靠近 RvB1; 而 INO80 的结构非常松散, 包含双层 12 元杂环的
RvB 模块, Arp8-Arp4-actin 远离 RvB1.

限于 Ino80 显著的结构柔性给三维重构带来的 严峻挑战, 已发表的 Ino80 结构信息采用了化学交联 进行了稳定, 并在冷冻制样时加入了重金属染液以 提高祄度; 所解析的结构不仅存在过度交联对构象 的显著影响, 而且重金属染液也不可避免地干扰构 象和稳定性. 系统优化了 INO80 和 SWR1 的生化制 备条件(尤其是轻度交联的条件), 将其完全包裹在玻 璃状态的冰中进行冷冻制样. 已重构真正意义上的 cryo-EM 结构与已报道的 cryo-负染的结构相比, 不 仅分辨率明显提高, 而且总体结构也显著不同. 此 外, 通过亚基缺失获得 INO80 和 SWR1 的一系列亚 复合物的三维结构, 这些亚复合物结构的细致比较, 有望获得 INO80 和 SWR1 各个亚基的清晰定位; 将 结构的信息与各个亚基的功能信息联系起来, 揭示 INO80 和 SWR1 复合体的模块架构及各个亚基的相 互作用关系.

中国科学技术大学蔡刚实验室瞄准染色质重塑 这一重大基本生物学问题, 专注利用冷冻电子显微 镜解析染色质重塑复合体及其与核小体的高分辨率 冷冻电子显微镜结构; 期望从功能和结构上阐明染 色质结构和基因组稳定性调控的分子机制, 尤其是 解析 SWI/SNF, INO80 和 SWR1 复合体及其与核小体 复合物的高分辨率冷冻电子显微镜结构, 清晰定位 各个复合体重要亚基和模块, 阐明这三种复合体重 塑核小体的分子机制.

\section{3 植物的表观遗传学}

与动物相似, 植物组蛋白 $\mathrm{H} 3$ 也存在保守的第四 位赖氨酸甲基化, 参与基因的转录激活功能. 在酵母 中, SET1 是唯的一个多梳家族蛋白质, 参与 COMPASS 复合体的形成, 具有使组蛋白 H3K4一、 二、三甲基化的功能. 而在拟南芥中, SET1 演化成 5 个蛋白, 分别为 ATX1, ATX2, ATX3, ATX4 及 ATX5 (图 6A). ATX1 与 ATX2 具有高度的同源性, 二者都 含有 PWWP, DAST, ePHD 及 SET 结构域, ATX1 具有 $\mathrm{H} 3 \mathrm{~K} 4$ 三甲基的功能, 而 ATX2 具有 $\mathrm{H} 3 \mathrm{~K} 4$ 二甲基功 能 ${ }^{[75]}$. 功能缺失的 $a t x 1$ 具有开花提前、影响花器官的 形成、降低干旱响应的表型 ${ }^{[76 ~ 79]}$, atx2 的功能缺失没 有任何的表型 ${ }^{[76]}$.

大规模转录组和组蛋白修饰的研究显示, 植物 
组蛋白 $\mathrm{H} 3 \mathrm{~K} 4 \mathrm{me} 3$ 与基因的转录水平呈正相关 ${ }^{[80,81]}$. 与动物不同, 植物中不存在可能直接结合 $\mathrm{H} 3 \mathrm{~K} 4 \mathrm{me} 3$ 的 TFIID 蛋白, 且 ATX1 的 ePHD 结构域与 PHD 不 同, 不具有结合 $\mathrm{H} 3 \mathrm{~K} 4 \mathrm{me} 3$ 的功能, 表明植物中组蛋白 H3K4me3 修饰, 不能形成一个正反馈调节转录的过 程, 可能存在不同于动物细胞中的机制. ATX1 在启动 子区和编码区, 有着两种不同的功能, 在启动子区参 与转录起始复合体的形成, 调控转录的开始; 在编码 区, 受到 Pol II 的招募, 完成甲基化 H3K4 的功能(图

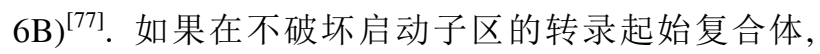
在只改变 ATX1 的甲基转移酶活性情况下, H3K4me3 则具有转录延伸的调控作用 ${ }^{[82,83]}$. H3K4me3 对基因 的转录正调控, 不仅体现在生长发育基因表达水平 的调控, 而且还体现在对环境诱导基因的调节, 并且 能在较长时间发挥功能. 将干旱处理的拟南芥, 恢复 后, 受干旱诱导的 $R D 29 B, R A B 18$ 等基因的表达水平 恢复至本底水平, 但 $\mathrm{H} 3 \mathrm{~K} 4 \mathrm{me} 3$ 的水平依然与诱导水 平一致. 如果再次干旱诱导, $R D 29 B, R A B 18$ 基因的 表达水平, 即表现出比第一次诱导高出数倍的水平, 这种现象在玉米中同样存在. $R D 29 B, R A B 18$ 这类基 因, 称为有“干旱记忆”功能的基因, $\mathrm{H} 3 \mathrm{~K} 4 \mathrm{me} 3$ 是这类 “干旱记忆”基因上的重要标志 ${ }^{[82,84,85]}$.

拟南芥中 ATX1 是植物中唯一研究较为清楚的 TRITHORAX 蛋白，但报道其直接调控的基因仅为 为数不多的几个, 如 FLC, LTP3, NCED3, WRKY7,
$A P 1$ 等 $[76 \sim 78,86,87]$. 与 MLL1 不同, 拟南芥中的 TRITHORAX 蛋白不具有 CXXC 结构域 (图 6C), 因 此 ATX1 如何作用于目标基因并不清楚. ATX1 可能 与一些转录因子相互作用, 由转录因子直接结合与 目标基因, 完成 H3K4me3 的功能. ATX1 中含有 PWWP 结构域, 动物细胞中的 PWWP 结构域, 具有 结合 DNA 及组蛋白 $\mathrm{H} 3 \mathrm{~K} 36 \mathrm{~m} 3$ 的功能. 因此, ATX1 也可能直接结合目标基因，从而完成甲基化修饰. 此 外, 植物的 H3K4me3 主要集中在转录起始后 $300 \mathrm{bp}$ 区，在启动子区分布较少，形成的原因依然不清 楚 ${ }^{[78,80]}$. 组蛋白 $\mathrm{H} 3 \mathrm{~K} 4 \mathrm{me} 3$ 不仅参与转录的调节, 同 样也受转录延伸的调控. 在动物细胞中, CDK9 能够 特异地磷酸化转录延伸因子 SPT5 的保守苏氨酸位 点, 而磷酸化苏氨酸的 SPT5 能够与 COMPASS-like 复合体中的亚基 RTF1 相互作用 ${ }^{[88]}$. 在酵母中, BUR2 是 CDK9 的同源蛋白, 能够磷酸化 SPT5 上保守的丝 氨酸和苏氨酸, 虽然丝氨酸和苏氨酸的磷酸化与 COMPASS 之间相互作用还未知, 但功能缺失性的 BUR2 和 SPT5 能够降低 H3K4me3 和 H3K36me3 的 修饰, 表明转录延伸因子不仅参与转录调控, 同时也 参与 $\mathrm{H} 3 \mathrm{~K} 4 \mathrm{me} 3$ 的组蛋白修饰 ${ }^{[89]}$. 在拟南芥中, SPT5 存在 2 个拷贝，是否有类似的功能还有待阐述. 在生 长发育和对环境适应的过程中, 植物有着自身的独 特性, 通过对植物表观修饰的研究, 将有助于对表观

\section{A}

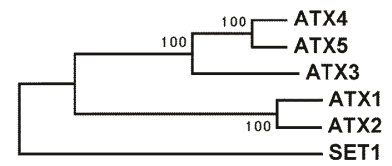

B

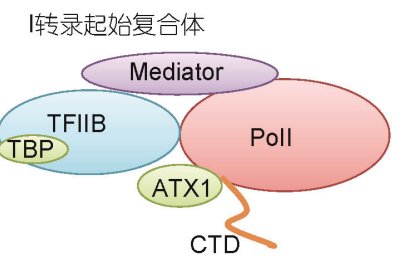

川转录起始

C

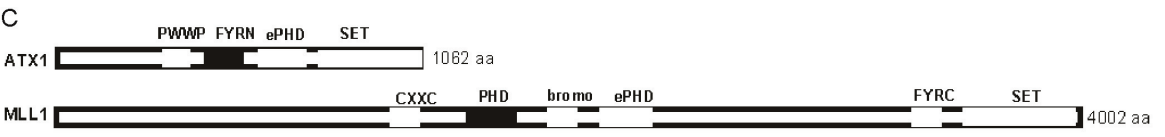

图 6 拟南芥 TRITHORAX 家族蛋白 ATX1 调控基因转录的过程

A: SET1 与拟南芥中 TRITHORAX 蛋白的进化树分析; B: ATX1 介导的 H3K4me3 与转录之间的关系. I 启动子区, ATX1 参与转录起始复合 体形成; II 在编码区 ATX1 受 Pol II 招募完成 H3K4me3; C: ATX1 和 MLL1 蛋白结构示意图 
遗传学的全面认识和理解.

\section{4 小结}

本综述基于中国科学技术大学生命科学学院的 成员近年来在表观遗传学方面的研究进展进行了回 顾, 包括了经典的表观遗传学修饰调控, 如组蛋白修
饰和 DNA 甲基化, 又加入了最新的 RNA 表观遗传学 和植物表观遗传学的研究内容, 从结构生物学和分 子生物学的角度回顾了研究进展. 表观遗传学作为 世界上转录调控的一个热点, 其进展日新月异. 相 信, 未来冷冻电子显微镜等新技术的进一步发展, 将 为表观遗传调控和染色质修饰相关的大复合物研究 提供更广阔的前景.

\section{参考文献}

1 Ullah M, Pelletier N, Xiao L, et al. Molecular architecture of quartet MOZ/MORF histone acetyltransferase complexes. Mol Cell Biol, 2008, 28: 6828-6843

2 Qiu Y, Liu L, Zhao C, et al. Combinatorial readout of unmodified H3R2 and acetylated H3K14 by the tandem PHD finger of MOZ reveals a regulatory mechanism for HOXA9 transcription. Gene Dev, 2012, 26: 1376-1391

3 Xiong X Z, Panchenko T, Yang S, et al. Selective recognition of histone crotonylation by double PHD fingers of MOZ and DPF2. Nat Chem Biol, 2016, 12: 1111

4 Qin S, Jin L, Zhang J H, et al. Recognition of unmodified histone H3 by the first PHD finger of Bromodomain-PHD finger protein 2 provides insights into the regulation of histone acetyltransferases monocytic leukemic zinc-finger protein (MOZ) and MOZ-related factor (MORF). J Biol Chem, 2011, 286: 36944-36955

5 Liu, L, Qin, S, Zhang, J H, et al., Solution structure of an atypical PHD finger in BRPF2 and its interaction with DNA.J Struct Biol,2012 180: $165-173$

6 Shen W Q, Xu C, Huang W, et al. Solution structure of human Brg1 bromodomain and its specific binding to acetylated histone tails. Biochemistry-Us, 2007, 46: 2100-2110

7 Huang $\mathrm{H} \mathrm{D}$, Zhang J H, Shen W Q, et al. Solution structure of the second bromodomain of Brd2 and its specific interaction with acetylated histone tails. Bmc Struct Biol, 2007, 7: 57

8 Liu Y, Wang X Q, Zhang J H, et al. Structural basis and binding properties of the second bromodomain of Brd4 with acetylated histone tails. Biochemistry-Us, 2008, 47: 6403-6417

9 Sun H B, Liu J X, Zhang J H, et al. Solution structure of BRD7 bromodomain and its interaction with acetylated peptides from histone H3 and H4. Biochem Bioph Res Co, 2007, 358: 435-441

10 Ng S S, Yue W W, Oppermann U, et al. Dynamic protein methylation in chromatin biology. Cell Mol Life Sci, 2009, 66: 407-422

11 Heintzman N D, Hon G C, Hawkins R D, et al. Histone modifications at human enhancers reflect global cell-type-specific gene expression. Nature, 2009, 459: 108-112

12 Santos-Rosa H, Schneider R, Bannister A J, et al. Active genes are tri-methylated at K4 of histone H3. Nature, 2002, 419: 407-411

13 Stewart M D, Li J W, Wong J M. Relationship between histone H3 lysine 9 methylation, transcription repression, and heterochromatin protein 1 recruitment. Mol Cell Biol, 2005, 25: 2525-2538

14 Li H T, Ilin S, Wang W K, et al. Molecular basis for site-specific read-out of histone H3K4me3 by the BPTF PHD finger of NURF. Nature, 2006, 442: 91-95

15 Xu C, Min J R. Structure and function of WD40 domain proteins. Protein Cell, 2011, 2: 202-214

16 Collins R E, Northrop J P, Horton J R, et al. The ankyrin repeats of G9a and GLP histone methyltransferases are mono- and dimethyllysine binding modules. Nat Struct Mol Biol, 2008, 15: 245-250

17 Yun M Y, Wu J, Workman J L, et al. Readers of histone modifications. Cell Res, 2011, 21: 564-578

18 Declais A C, Lilley D M J. New insight into the recognition of branched DNA structure by junction-resolving enzymes. Curr Opin Struc Biol, 2008, 18: 86-95

19 Samara N L, Wolberger C. A new chapter in the transcription SAGA. Curr Opin Struc Biol, 2011, 21: 767-774

20 Bian C B, Xu C, Ruan J B, et al. Sgf29 binds histone H3K4me2/3 and is required for SAGA complex recruitment and histone H3 acetylation. Embo J, 2011, 30: 2829-2842

21 Mosammaparast N, Shi Y. Reversal of histone methylation: biochemical and molecular mechanisms of histone demethylases. Annu Rev Biochem, 2010, 79: 155-179 
24 Shi Y, Whetstine J R. Dynamic regulation of histone lysine methylation by demethylases. Mol Cell, 2007, 25: 1-14

25 Klose R J, Yi Z. Regulation of histone methylation by demethylimination and demethylation. Nat Rev Mol Cell Bio, 2007, 8: 307-318

26 Eilbracht J, Reichenzeller M, Hergt M, et al. NO66, a highly conserved dual location protein in the nucleolus and in a special type of synchronously replicating chromatin. Mol Biol Cell, 2004, 15: 1816-1832

27 Tao Y, Wu M H, Zhou X, et al. Structural insights into histone demethylase NO66 in interaction with osteoblast-specific transcription factor osterix and gene repression. J Biol Chem, 2013, 288: 16430-16437

28 Wang C L, Zhang Q D, Hang T R, et al. Structure of the JmjC domain-containing protein NO66 complexed with ribosomal protein Rpl8. Acta Crystallogr D, 2015, 71: 1955-1964

29 Chowdhury R, Sekirnik R, Brissett N C, et al. Ribosomal oxygenases are structurally conserved from prokaryotes to humans. Nature, 2014, 510: 422-426

30 Sinha K M, Yasuda H, Zhou X, et al. Osterix and NO66 histone demethylase control the chromatin of osterix target genes during osteoblast differentiation. J Bone Miner Res, 2014, 29: 855-865

31 Bedford M T, Richard S. Arginine methylation: an emerging regulator of protein function. Mol Cell, 2005, 18: 263-272

32 Bedford M T, Clarke S G. Protein arginine methylation in mammals: who, what, and why. Mol Cell, 2009, 33: 1-13

33 Di Lorenzo A, Bedford M T. Histone arginine methylation. Febs Lett, 2011, 585: 2024-2031

34 Wang C Y, Zhu Y W, Chen J J, et al. Crystal structure of arginine methyltransferase 6 from Trypanosoma brucei. PLoS One, 2014, 9: e87267

35 Wang C Y, Zhu Y W, Caceres T B, et al. Structural determinants for the strict monomethylation activity by Trypanosoma brucei protein arginine methyltransferase 7. Structure, 2014, 22: 756-768

36 Liu K, Chen C, Guo Y H, et al. Structural basis for recognition of arginine methylated Piwi proteins by the extended Tudor domain. Proc Natl Acad Sci USA, 2010, 107: 18398-18403

37 Wade P A. Methyl CpG binding proteins: coupling chromatin architecture to gene regulation. Oncogene, 2001, 20: 3166-3173

38 Allen M D, Grummitt C G, Hilcenko C, et al. Solution structure of the nonmethyl-CpG-binding CXXC domain of the leukaemia-associated MLL histone methyltransferase. Embo J, 2006, 25: 4503-4512

39 Long H K, Blackledge N P, Klose R J. ZF-CxxC domain-containing proteins, CpG islands and the chromatin connection. Biochem Soc T, 2013, 41: 727-740

40 Xu C, Bian C B, Lam R, et al. The structural basis for selective binding of non-methylated CpG islands by the CFP1 CXXC domain. Nat Commun, 2011, 2: 385-396

41 Thomson J P, Skene P J, Selfridge J, et al. CpG islands influence chromatin structure via the CpG-binding protein Cfp1. Nature, 2010, 464: U1082-U1162

$42 \mathrm{Xu} \mathrm{Y} \mathrm{F,} \mathrm{Xu} \mathrm{C,} \mathrm{Kato} \mathrm{A,} \mathrm{et} \mathrm{al.} \mathrm{Tet3} \mathrm{CXXC} \mathrm{Domain} \mathrm{and} \mathrm{dioxygenase} \mathrm{activity} \mathrm{cooperatively} \mathrm{regulate} \mathrm{key} \mathrm{genes} \mathrm{for} \mathrm{xenopus} \mathrm{eye} \mathrm{and} \mathrm{neural}$ development. Cell, 2012, 151: 1200-1213

43 Rozenski J, Crain P F, McCloskey J A. The RNA modification database: 1999 update. Nucleic Acids Res, 1999, 27: 196-197

44 Fu Y, Dominissini D, Rechavi G, et al. Gene expression regulation mediated through reversible m(6)A RNA methylation. Nat Rev Genet, 2014, 15: 293-306

45 Wang Y, Li Y, Toth J I, et al. N-6-methyladenosine modification destabilizes developmental regulators in embryonic stem cells. Nat Cell Biol, 2014, 16: 191-198

46 Liu J Z, Yue Y N, Han D L, et al. A METTL3-METTL14 complex mediates mammalian nuclear RNA N-6-adenosine methylation. Nat Chem Biol, 2014, 10: 93-95

47 Ping X L, Sun B F, Wang L, et al. Mammalian WTAP is a regulatory subunit of the RNA N6-methyladenosine methyltransferase. Cell Res, 2014, 24: 177-189

48 Jia G F, Fu Y, Zhao X, et al. N6-Methyladenosine in nuclear RNA is a major substrate of the obesity-associated FTO. Nat Chem Biol, 2011, 7: 885-887

49 Zheng G Q, Dahl J A, Niu Y M, et al. ALKBH5 is a mammalian RNA demethylase that impacts rna metabolism and mouse fertility. Mol Cell, 2013, 49: 18-29

50 Dominissini D, Moshitch-Moshkovitz S, Schwartz S, et al. Topology of the human and mouse m(6)A RNA methylomes revealed by 
m(6)A-seq. Nature, 2012, 485: U201-U284

51 Wang X, Lu Z K, Gomez A, et al. N-6-methyladenosine-dependent regulation of messenger RNA stability. Nature, 2014, 505: 117-120

52 Xu C, Wang X, Liu K, et al. Structural basis for selective binding of m(6)A RNA by the YTHDC1 YTH domain. Nat Chem Biol, 2014, 10: $927-929$

53 Luo S K, Tong L. Molecular basis for the recognition of methylated adenines in RNA by the eukaryotic YTH domain. Proc Natl Acad Sci USA, 2014, 111: 13834-13839

54 Li F D, Zhao D B, Wu J H, et al. Structure of the YTH domain of human YTHDF2 in complex with an m(6)A mononucleotide reveals an aromatic cage for m(6)A recognition. Cell Res, 2014, 24: 1490-1492

55 Xu C, Liu K, Ahmed H, et al. Structural basis for the discriminative recognition of N-6-Methyladenosine RNA by the human YT521-B homology domain family of proteins. J Biol Chem, 2015, 290: 24902-24913

56 Aik W, Scotti J S, Choi H, et al. Structure of human RNA N-6-methyladenine demethylase ALKBH5 provides insights into its mechanisms of nucleic acid recognition and demethylation. Nucleic Acids Res, 2014, 42: 4741-4754

57 Xu C, Liu K, Tempel W, et al. Structures of human ALKBH5 demethylase reveal a unique binding mode for specific single-stranded N-6-Methyladenosine RNA demethylation. J Biol Chem, 2014, 289: 17299-17311

58 Sudarsanam P, Winston F. The Swi/Snf family-nucleosome-remodeling complexes and transcriptional control. Trends Genet, 2000, 16: 345-351

59 Conaway R C, Conaway J W. The INO80 chromatin remodeling complex in transcription, replication and repair. Trends Biochem Sci, 2009, 34: 71-77

60 Kasten M M, Clapier C R, Cairns B R. SnapShot: chromatin remodeling: SWI/SNF. Cell, 2011, 144: 310

61 Narlikar G J, Sundaramoorthy R, Owen-Hughes T. Mechanisms and functions of ATP-Dependent chromatin-remodeling enzymes. Cell, 2013, 154: 490-503

62 Clapier C R, Cairns B R. The biology of chromatin remodeling complexes. Annu Rev Biochem, 2009, 78: 273-304

63 Yang X F, Zaurin R, Beato M, et al. Swi3p controls SWI/SNF assembly and ATP-dependent H2A-H2B displacement. Nat Struct Mol Biol, 2007, 14: 540-547

64 Swanson M J, Qiu H F, Sumibcay L, et al. A multiplicity of coactivators is required by Gen 4p at individual promoters in vivo. Mol Cell Biol, 2003, 23: 2800-2820

65 Ferreira M E, Prochasson P, Berndt K D, et al. Activator-binding domains of the SWI/SNF chromatin remodeling complex characterized in vitro are required for its recruitment to promoters in vivo. FEBS J, 2009, 276: 2557-2565

66 Ho L, Crabtree G R. Chromatin remodelling during development. Nature, 2010, 463: 474-484

67 Helming K C, Wang X F, Roberts C W M. Vulnerabilities of mutant SWI/SNF complexes in cancer. Cancer Cell, 2014, 26: 309-317

68 Bitler B G, Fatkhutdinov N, Zhang R G. Potential therapeutic targets in ARID1A-mutated cancers. Expert Opin Ther Tar, 2015, 19: $1419-1422$

69 Biegel J A, Busse T M, Weissman B E. SWI/SNF chromatin remodeling complexes and cancer. Am J Med Genet C, 2014, 166: 350-366

70 Leschziner A E, Nogales E. Visualizing flexibility at molecular resolution: Analysis of heterogeneity in single-particle electron microscopy reconstructions. Annu Rev Bioph Biom, 2007, 36: 43-62

71 Bao Y H, Shen X T. SnapShot: chromatin remodeling: INO80 and SWR1. Cell, 2011, 144: U158-U165

72 Gerhold C B, Gasser S M. INO80 and SWR complexes: relating structure to function in chromatin remodeling. Trends Cell Biol, 2014, 24: $619-631$

73 Nguyen V Q, Ranjan A, Stengel F, et al. Molecular architecture of the ATP-dependent chromatin-remodeling complex SWR1. Cell, 2013, 154: 1220-1231

74 Tosi A, Haas C, Herzog F, et al. Structure and subunit topology of the INO80 chromatin remodeler and its nucleosome complex. Cell, 2013, 154: 1207-1219

75 Saleh A, Alvarez-Venegas R, Yilmaz M, et al. The highly similar Arabidopsis homologs of trithorax ATX1 and ATX2 encode proteins with divergent biochemical functions. Plant Cell, 2008, 20: 568-579

76 Saleh A, Alvarez-Venegas R, Avramova Z. Dynamic and stable histone H3 methylation patterns at the Arabidopsis FLC and AP1 loci. Gene, 2008, 423: 43-47

77 Ding Y, Avramova Z, Fromm M. Two distinct roles of Arabidopsis homolog of trithorax1 (ATX1) at promoters and within transcribed regions of ATX1-regulated Genes. Plant Cell, 2011, 23: 350-363

78 Ding Y, Avramova Z, Fromm M. The Arabidopsis trithorax-like factor ATX1 functions in dehydration stress responses via 
ABA-dependent and ABA-independent pathways. Plant J, 2011, 66: 735-744

79 Ding Y, Fromm M, Avramova Z. Multiple exposures to drought 'train' transcriptional responses in Arabidopsis. Nat Commun, 2012, 3 : 740

80 van Dijk K, Ding Y, Malkaram S, et al. Dynamic changes in genome-wide histone H3 lysine 4 methylation patterns in response to dehydration stress in Arabidopsis thaliana. Bmc Plant Biol, 2010, 10: 238

81 Zhang X Y, Bernatavichute Y V, Cokus S, et al. Genome-wide analysis of mono-, di- and trimethylation of histone $\mathrm{H} 3$ lysine 4 in Arabidopsis thaliana. Genome Biol, 2009, 10: 100-105

82 Ding Y, Ndamukong I, Xu Z S, et al. ATX1-generated H3K4me3 is required for efficient elongation of transcription, not initiation, at ATX1-regulated genes. PLoS Genet, 2012, 8: 1208-1213

83 Fromm M, Avramova Z. ATX1/AtCOMPASS and the H3K4me3 marks: how do they activate Arabidopsis genes? Curr Opin Plant Biol, 2014, 21: 75-82

84 Ding Y, Virlouvet L, Liu N, et al. Dehydration stress memory genes of Zea mays comparison with Arabidopsis thaliana. Bmc Plant Biol, 2014, 14: 1-15

85 Ding Y, Liu N, Virlouvet L, et al. Four distinct types of dehydration stress memory genes in Arabidopsis thaliana. Bmc Plant Biol, 2013, 13: 229

86 Pien S, Fleury D, Mylne J S, et al. ARABIDOPSIS TRITHORAX1 dynamically regulates FLOWERING LOCUS C activation via histone 3 lysine 4 trimethylation. Plant Cell, 2008, 20: 580-588

87 Alvarez-Venegas R, Pien S, Sadder M, et al. ATX-1, an Arabidopsis homolog of trithorax, activates flower homeotic genes. Curr Biol, 2003, 13: 627-637

88 Wier A D, Mayekar M K, Héroux A, et al. Structural basis for Spt5-mediated recruitment of the Paf1 complex to chromatin. Proc Natl Acad Sci USA, 2013, 110: 17290-17295

89 Zhou K, Kuo W H W, Fillingham J, et al. Control of transcriptional elongation and cotranscriptional histone modification by the yeast BUR kinase substrate Spt5. Proc Natl Acad Sci USA, 2009, 106: 6956-6961 


\title{
Recent progress in epigenetics
}

\author{
DING Yong, XU Chao, WU JiHui, SHI YunYu, ZANG JianYe \& CAI Gang \\ School of Life Sciences, University of Science and Technology of China, Hefei 230027, China
}

The regulation of eukaryotic gene expression by epigenetic mechanisms has been a hot topic in the biological research field. Recently, Chinese researchers have made major progress in the field of epigenetics. This article reviews three aspects of the work of researchers in the School of Life of Sciences, University of Science and Technology of China, i.e., epigenetic marks (including RNA epigenetics), chromatin remodeling, and plant epigenetics.

epigenetics, histone modifications, CXXC domain, royal family, DNA methylation, RNA methylation, chromatin remodeling

doi: 10.1360/N052016-00317 